OPEN ACCESS

Edited by:

Chris Greening,

Monash University, Australia

Reviewed by:

Bat-Erdene Jugder,

Harvard Medical School,

United States

Rhiannon Mari Evans,

University of Oxford, United Kingdom

*Correspondence:

Constanze Pinske

constanze.pinske@

mikrobiologie.uni-halle.de

R. Gary Sawers

gary.sawers@mikrobiologie.

uni-halle.de

Specialty section:

This article was submitted to Microbial Physiology and Metabolism,

a section of the journal

Frontiers in Microbiology

Received: 19 September 2018

Accepted: 05 November 2018

Published: 21 November 2018

Citation:

Dragomirova N, Rothe $P$ Schwoch S, Hartwig S, Pinske $C$ and Sawers RG (2018) Insights Into the Redox Sensitivity of Chloroflexi

Hup-Hydrogenase Derived From

Studies in Escherichia coli: Merits and Pitfalls of Heterologous [NiFe]-Hydrogenase Synthesis.

Front. Microbiol. 9:2837. doi: 10.3389/fmicb.2018.02837

\section{Insights Into the Redox Sensitivity of Chloroflexi Hup-Hydrogenase Derived From Studies in Escherichia coli: Merits and Pitfalls of Heterologous [NiFe]-Hydrogenase Synthesis}

\author{
Nadya Dragomirova, Patricia Rothe, Stefan Schwoch, Stefanie Hartwig, \\ Constanze Pinske* and R. Gary Sawers*
}

Institute of Microbiology, Martin-Luther University Halle-Wittenberg, Halle, Germany

The highly oxygen-sensitive hydrogen uptake (Hup) hydrogenase from Dehalococcoides mccartyi forms part of a protein-based respiratory chain coupling hydrogen oxidation with organohalide reduction on the outside of the cell. The HupXSL proteins were previously shown to be synthesized and enzymatically active in Escherichia coli. Here we examined the growth conditions that deliver active Hup enzyme that couples $\mathrm{H}_{2}$ oxidation to benzyl viologen (BV) reduction, and identified host factors important for this process. In a genetic background lacking the three main hydrogenases of $E$. coli we could show that additional deletion of genes necessary for selenocysteine biosynthesis resulted in inactive Hup enzyme, suggesting requirement of a formate dehydrogenase for Hup activity. Hup activity proved to be dependent on the presence of formate dehydrogenase $(\mathrm{Fdh}-\mathrm{H})$, which is typically associated with the $\mathrm{H}_{2}$-evolving formate hydrogenlyase $(\mathrm{FHL})$ complex in the cytoplasm. Further analyses revealed that heterologous Hup activity could be recovered if the genes encoding the ferredoxin-like electron-transfer protein HupX, as well as the related $\mathrm{HycB}$ small subunit of Fdh-H were also deleted. These findings indicated that the catalytic HupL and electron-transferring HupS subunits were sufficient for enzyme activity with BV. The presence of the HupX or HycB proteins in the absence of $\mathrm{Fdh}-\mathrm{H}$ therefore appears to cause inactivation of the HupSL enzyme. This is possibly because HupX or HycB aided transfer of electrons to the quinone pool or other oxidoreductase complexes, thus maintaining the HupSL heterodimer in a continuously oxidized state causing its inactivation. This proposal was supported by the observation that growth under either aerobic or anaerobic respiratory conditions did not yield an active HupSL. These studies thus provide a system to understand the redox sensitivity of this heterologously synthesized hydrogenase.

Keywords: hydrogen, formate, ferredoxin-like proteins, electron transfer, uptake hydrogenase, heterologous expression 


\section{INTRODUCTION}

The bacterial genus Dehalococcoides belongs to the phylum Chloroflexi and the type species D. mccartyi is completely dependent on hydrogen for growth (Löffler et al., 2013; Schubert et al., 2018). D. mccartyi synthesizes several types of [NiFe]-hydrogenase (Hyd), and the hydrogen-uptake (Hup) hydrogenase is thought to be the main enzyme involved in $\mathrm{H}_{2}$-driven organohalide respiration. As D. mccartyi lacks quinones (Kube et al., 2005; Schipp et al., 2013), a direct transfer of the electrons derived from $\mathrm{H}_{2}$ oxidation by Hup via protein-protein interaction has been implicated (Kublik et al., 2016; Hartwig et al., 2017; Seidel et al., 2018). The Hup enzyme is found in a respiratory supercomplex comprising a two-subunit complex iron-sulfur molybdoprotein, OmeAB (organohalide molybdoenzyme) and one of a number of reductive dehalogenases (Rdh), which catalyze the reduction of particular organohalides that function as electron acceptors for the bacterium (Fincker and Spormann, 2017; Schubert et al., 2018). In addition, the ferredoxin-like protein HupX, which resembles electron-transferring subunits of oxidoreductases, is associated with the complex.

Hup comprises two structural components: the catalytic subunit HupL, containing the $\mathrm{NiFe}(\mathrm{CN})_{2} \mathrm{CO}$ cofactor and HupS, the small electron-transferring subunit, which is predicted to have three iron-sulfur clusters. The membrane-associated, ferredoxin-like protein HupX is encoded within the operon of the Hup hydrogenase, but seems to associate more tightly with the core OmeAB-Rdh complex (Hartwig et al., 2017; Seidel et al., 2018), suggesting that it is the main mediator of electron transfer and acts as a "connector" protein between HupSL and the rest of the complex. HupX is homologous to HybA, a component of the Hyd-2 $\mathrm{H}_{2}$-oxidizing hydrogenase of Escherichia coli (Sargent et al., 1998; Beaton et al., 2018) and recent studies have provided strong evidence indicating that $\mathrm{HybA}$ is responsible for coupling electron transfer to the quinone pool, as Hyd-2 has no membrane subunit with a recognized heme cofactor, necessary for electron transfer into the membrane (Dubini et al., 2002; Pinske et al., 2015; Beaton et al., 2018).

The ferredoxin-like family of electron-transfer proteins harbors four [4Fe-4S] clusters and an interaction network of several members of this family has been uncovered recently in E. coli (Pinske, 2018). One member is $\mathrm{HycB}$, the small subunit of the formate dehydrogenase (Fdh-H) that forms one of the two catalytic centers of the formate hydrogenlyase (FHL) complex, and another is the related protein HydN, which is proposed to be involved in FHL complex assembly (Pinske, 2018). Generally, however, the physiological function of most members of this emerging superfamily of iron-sulfur-containing electron transfer proteins is not understood.

Due to the fact that D. mccartyi grows extremely slowly and produces limited amounts of biomass, making biochemical studies challenging, we have established a heterologous expression system for the synthesis of a functional Hup enzyme in E. coli (Hartwig et al., 2015b). It is hoped that this system will facilitate a detailed biochemical characterization of Hup. Despite the significant phylogenetic distance between D. mccartyi and E. coli, the Hyp maturation system responsible for [NiFe]-cofactor biosynthesis and insertion (Böck et al., 2006) is capable of recognizing the HupL apoprotein and generating an active enzyme when the complete operon encoding Hup is expressed under anaerobic conditions (Hartwig et al., 2015b).

As well as the three structural genes, the hupXSLhoxM operon (Figure 1A) also encodes a HupL-specific maturation endoprotease (HoxM). Initial characterization of the heterologously synthesized Hup enzyme identified a fast-migrating complex, mainly comprising HupS and HupL after native-PAGE, which migrated at a similar position as the complex present in crude extracts of D. mccartyi that contained HupSL and minor amounts of HupX (Hartwig et al., 2015b). This suggests that HupSL alone is capable of catalyzing $\mathrm{H}_{2}$-dependent reduction of the redox dye $\mathrm{BV}$. The activity of the complex was oxygen-sensitive, even when synthesized anaerobically in the heterologous host (Hartwig et al., 2015b), suggesting that a cofactor in the enzyme is redox-sensitive. Whether this redox-sensitive cofactor is in HupL, HupS or HupX is unclear. Therefore, to address these questions, in the current study we decided to determine the conditions necessary for heterologous production of HupSL activity and whether any other components of the host's metabolism, other than the Hyp proteins, are required for activity to be visualized. Surprisingly, we found a strong dependence for HupSL activity on the Fdh-H enzyme of the FHL complex. This dependence on Fdh-H for activity proved to be linked to an involvement of ferredoxin-like electron transfer proteins and to the redox sensitivity of the HupSL heterodimer.

\section{MATERIALS AND METHODS}

\section{Strains and Growth Conditions}

The strains listed in Table 1 were used in this study. For routine molecular biology studies, growth was on LB-agar plates or in LBbroth at $37^{\circ} \mathrm{C}$ (Miller, 1972). Anaerobic growths were performed at $37^{\circ} \mathrm{C}$ as standing liquid cultures and cells were usually grown in $\mathrm{M} 9$ minimal medium $\left(47.6 \mathrm{mM} \mathrm{Na}_{2} \mathrm{HPO}_{4} \times 2 \mathrm{H}_{2} \mathrm{O}, 22 \mathrm{mM}\right.$ $\mathrm{KH}_{2} \mathrm{PO}_{4}, 8.4 \mathrm{mM} \mathrm{NaCl}, 20 \mathrm{mM} \mathrm{NH}_{4} \mathrm{Cl}, 2 \mathrm{mM} \mathrm{MgSO}_{4}, 0.1 \mathrm{mM}$ $\mathrm{CaCl}_{2}, 0.1 \mathrm{mM}$ thiamin dichloride, $0.2 \% \mathrm{w} / \mathrm{v}$ casamino acids) containing $0.8 \%(\mathrm{w} / \mathrm{v})$ glucose, or $0.4 \%(\mathrm{v} / \mathrm{v})$ glycerol plus 15 $\mathrm{mM}$ fumarate, or $0.8 \%(\mathrm{w} / \mathrm{v})$ glucose plus $1 \%(\mathrm{w} / \mathrm{v})$ nitrate, where indicated, as described (Sambrook et al., 1989). When growth in rich medium was performed, buffered TGYEP (1\% $\mathrm{w} / \mathrm{v}$ tryptone, $0.5 \% \mathrm{w} / \mathrm{v}$ yeast extract, $0.8 \% \mathrm{w} / \mathrm{v}$ glucose, $100 \mathrm{mM}$ potassium phosphate, $\mathrm{pH}$ 6.5) was used (Begg et al., 1977). The growth medium was supplemented with trace element solution SLA (Hormann and Andreesen, 1989). When required, the antibiotic kanamycin or chloramphenicol was added to a final concentration of 50 or $25 \mu \mathrm{g} \mathrm{ml}{ }^{-1}$, respectively. Cells were harvested anaerobically by centrifugation at $5,000 \mathrm{~g}$ for $15 \mathrm{~min}$ at $4^{\circ} \mathrm{C}$ when cultures had reached an $\mathrm{OD}_{600} \mathrm{~nm}$ of between 0.8 and 1.2. Cell pellets were used immediately or stored at $-20^{\circ} \mathrm{C}$ until use. 
$\mathbf{A}$
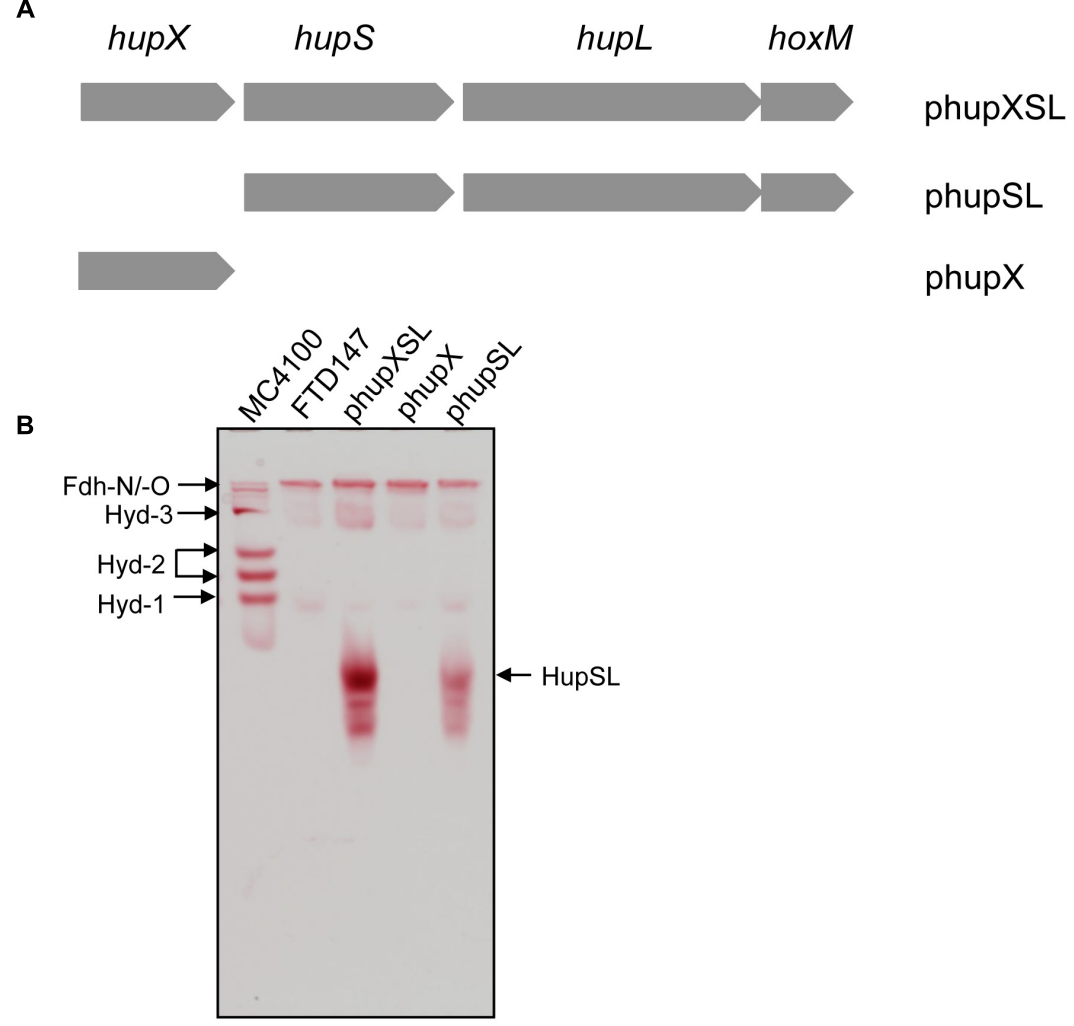

FIGURE 1 | HupX is not required for heterologous HupSL activity. (A) Schematic representation of the plasmids used in this study is shown. The plasmid inserts are not drawn to scale but the complete hupXSLhoxM region encompasses 4216 bp (Hartwig et al., 2015b). (B) An in-gel activity stain for hydrogen-oxidizing activity is shown. Crude extracts ( $70 \mu \mathrm{g}$ of protein; $30 \mu \mathrm{g}$ in the case of wild type MC4100) derived from FTD147 ( $\Delta$ hyaB $\Delta$ hybC $\Delta$ hycE) carrying the indicated plasmids were applied to a native polyacrylamide gel ( $7.5 \% \mathrm{w} / \mathrm{v}$ polyacrylamide). The migration positions of HupSL and the $E$. coli hydrogenases are indicated. The formate dehydrogenases Fdh-N and Fdh-O (Fdh-N/O) have a weak hydrogen-oxidizing activity (Soboh et al., 2011), which is also indicated and was used as an internal loading control for the experiment.

\section{Plasmid and Strain Construction}

The hupX gene (cbdbA131) was amplified as a 1212 bp DNA fragment from chromosomal DNA isolated from D. mccartyi strain CBDB1 using Pfu DNA polymerase and the oligonucleotides hupX_fw (5' GGGGCATATGCCTAATGGAATGCTGATTG- ${ }^{\prime}$ ) - and hupX_re (5'-GGGGCTCGAGCTAGTGCTTGCCAGCCTTG$3^{\prime}$ ) and cloned in plasmid pACYC-Duet-I. Plasmid phupSL was constructed by using pSHH18 (referred to as phupXSL throughout this study) as template in a PCR mutagenesis employing the ${ }^{\circledR}{ }^{\circledast}$ Site-Directed Mutagenesis Kit (New England Biolabs, NEB). Care was taken when deleting the hupX gene to ensure that the ribosome binding site for the downstream hupS gene remained intact by using the oligonucleotides hupSLhoxM_fw (5'-ATGGAGTAGGAAAAATGTTTAATAC$\left.3^{\prime}\right)$ and hupSLhoxM_re (5'-TCCTGTTGCCCCCCTTGT-3') and by following the instructions given in the Q $5{ }^{\circledR}$ Site-Directed Mutagenesis Kit.

E. coli strains were constructed using P1kc-mediated phage transduction (Miller, 1972) to introduce the respective defined deletion mutation from the appropriate donor strain obtained from the Keio collection (Baba et al., 2006) to generate the series of FTD147 mutants lacking the structural genes encoding the three formate dehydrogenases of E. coli. When multiple gene knock-outs were constructed, the plasmid pCP20 was used to remove the kanamycin antibiotic resistance cassette as described (Cherepanov and Wackernagel, 1995).

\section{Preparation of Crude Cell Extracts and Cell Fractionation}

Unless otherwise stated, all experiments were performed in an anaerobic $\mathrm{Coy}^{\mathrm{TM}}$ chamber under an atmosphere of $95 \%$ nitrogen $/ 5 \%$ hydrogen. For standard hydrogenase enzyme activity determination, E. coli cell paste was re-suspended at a ratio of $1 \mathrm{~g}$ cell wet weight to $3 \mathrm{ml} 50 \mathrm{mM}$ MOPS buffer, $\mathrm{pH} 7$. Cells were disrupted by sonication $(30 \mathrm{~W}$ power for $5 \mathrm{~min}$ with $0.5 \mathrm{~s}$ pulses). Unbroken cells and cell debris were removed by centrifugation for $30 \mathrm{~min}$ at $50,000 \mathrm{~g}$ and $4^{\circ} \mathrm{C}$. The resulting crude extract, unless otherwise stated, was used for all studies reported herein.

In order to perform sub-cellular fractionation, periplasmic, soluble and membrane fractions were isolated as described (Sawers et al., 1985). 
TABLE 1 | Strains and plasmids used in this study.

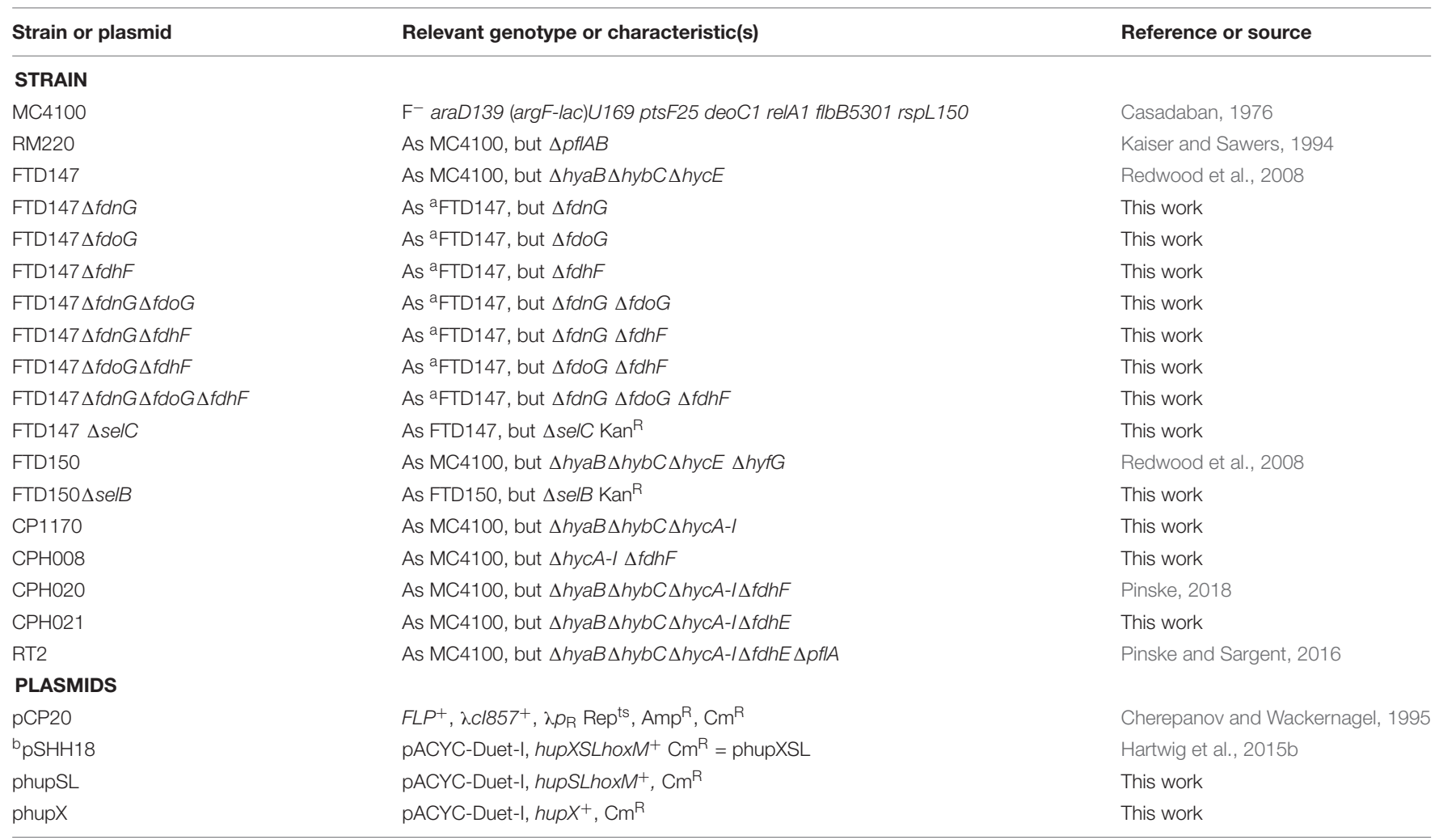

The series of FTD147 mutants with different combinations of Fdh gene mutations was constructed by transduction of mutations from the corresponding Keio collection of mutants (see Materials and Methods). ${ }^{b}$ Note that for reasons of clarity, this plasmid was referred to as phupXSL throughout this study.

Determination of protein concentration was done as described (Lowry et al., 1951).

\section{Non-denaturing Polyacrylamide Gel Electrophoresis and Activity-Staining}

Unless otherwise specified, non-denaturing polyacrylamide gel electrophoresis (PAGE) was performed anaerobically. Separating gels included $0.1 \%(\mathrm{w} / \mathrm{v})$ Triton X-100 as described (Ballantine and Boxer, 1985). The crude extracts, or sub-cellular fractions, were incubated with a final concentration of $4 \%(\mathrm{w} / \mathrm{v})$ Triton $\mathrm{X}-100$ prior to application (usually $50 \mu \mathrm{g}$ of protein) to the gel, which included $6 \%(\mathrm{w} / \mathrm{v})$ polyacrylamide. Hydrogenase activitystaining was done in $50 \mathrm{mM}$ MOPS buffer $\mathrm{pH} 7.0$, as described (Sawers et al., 1985; Pinske et al., 2012), and included 0.5 mM BV and $1 \mathrm{mM} \mathrm{2,3,5-triphenyltetrazolium} \mathrm{chloride} \mathrm{(TTC).} \mathrm{Gels} \mathrm{were}$ incubated under an atmosphere of $100 \%$ hydrogen gas.

\section{Hydrogenase Activity Assay}

Measurement of hydrogenase enzyme activity using BV as electron acceptor was performed as described (Ballantine and Boxer, 1985; Pinske et al., 2011). Briefly, anaerobically prepared cuvettes $(1.6 \mathrm{ml})$ were filled with $0.8 \mathrm{ml}$ of $\mathrm{H}_{2}$-saturated, anaerobic $50 \mathrm{mM}$ MOPS buffer, $\mathrm{pH} 7.0$, including $4 \mathrm{mM}$ BV and placed under $\mathrm{a}_{2}$ atmosphere. After baseline determination, the assay was initiated by adding enzyme sample (approximately $150 \mu \mathrm{g}$ of protein). All assays were performed at $25^{\circ} \mathrm{C}$. The wavelength used was $600 \mathrm{~nm}$ and an $\varepsilon_{\mathrm{M}}$ value of $7400 \mathrm{M}^{-1} \mathrm{~cm}^{-1}$ was assumed for reduced BV. One million unit of enzyme activity corresponded to the reduction of $1 \mathrm{nmol}$ of substrate $\mathrm{min}^{-1}$. Enzyme assays were performed in triplicate using three biological replicates.

\section{Denaturing Polyacrylamide Gel Electrophoresis (PAGE) and Western Blotting}

Polypeptides in crude extracts were separated by $12.5 \%(\mathrm{w} / \mathrm{v})$ sodium dodecyl sulfate (SDS)-PAGE (Laemmli, 1970) and gels were either stained with Coomassie Brilliant Blue R or transferred to nitrocellulose membranes for western blotting, which was performed as described (Towbin et al., 1979). The antibodies used were either anti-Strep-tag (IBA Life Sciences), anti-Hyd-2 (Sargent et al., 1998), anti-HupL or anti-HupX peptide antibodies (Hartwig et al., 2017).

\section{RESULTS}

\section{HupL and HupS Are Sufficient for BV Reduction Activity}

The hupXSLhoxM operon has been shown to be functional in anaerobically grown E. coli (Hartwig et al., 2015b). In order to determine whether all three structural components 
(HupSL and HupX) are essential for the manifestation of the $\mathrm{H}_{2}$ :BV oxidoreductase activity observed in that study, we constructed two additional plasmid derivatives, one of which carried only the hupX gene, while the other included hupSLhoxM but lacked hupX (Figure 1A). These plasmids, along with pSHH18 (hupXSLhoxM ${ }^{+}$; Hartwig et al., 2015b; here referred to as phupXSL in the aid of clarity), were introduced into FTD147, which lacks the genes encoding the catalytic subunits of Hyd-1, Hyd-2, and Hyd-3 (Redwood et al., 2008). After fermentative growth, crude extracts were separated in nativePAGE and stained for hydrogenase enzyme activity (Figure 1B). As anticipated, the plasmid encoding only HupX showed no hydrogenase enzyme activity in extracts of strain FTD147 ( $\triangle h y a B \Delta h y b C \Delta h y c E$ ), while both of the other plasmids resulted in a fast-migrating activity band corresponding to the HupSL heterodimer (Figure 1B). Notably, although the activity resulting from introduction of the plasmid lacking the hupX gene (phupSL in Figure 1B) was apparently weaker than that resulting from introduction of phupXSL, both enzyme activities showed very similar migration characteristics, indicating that HupX is neither necessary for the ability of the enzyme to reduce BV nor seems to co-migrate with HupSL in this particular activity band. This result correlates well with earlier mass spectrometric analyses of heterologously expressed enzyme, which identified mainly the HupL protein (Hartwig et al., 2015b).

\section{Manifestation of Heterologous HupSL Enzyme Activity Requires Fermentative Growth Conditions}

In order to optimize conditions for the analysis of heterologously produced HupSL activity, we tested different anaerobic growth conditions using FTD147 ( $\triangle h y a B \Delta h y b C \Delta h y c E)$ transformed with either phupXSL or phupSL (Figure 2A). The activity band was slightly more intense when cells were grown with $0.8 \% \mathrm{w} / \mathrm{v}$ glucose compared with half that glucose concentration $(0.4 \%$ w/v). Suprisingly, however, no HupSL activity could be detected when cells were grown under anaerobic respiratory conditions, with either glycerol and fumarate or glucose and nitrate. Western blot analysis of the extracts derived from anaerobically grown strains after separation by SDS-PAGE using peptide antibodies raised against HupL revealed that the HupL polypeptide could be detected in each extract (Figure 2B). This indicates that a lack of transcription of the hup genes under respiratory conditions was not the reason for absence of HupSL enzyme activity. Surprisingly, it was not possible to restore in vitro HupSL enzyme activity to these extracts, even by incubating the extracts under reducing conditions. This suggests that the HupSL enzyme was irreversibly inhibited under the oxidizing conditions that prevailed within the cells grown under these conditions.

Quantitative assessment of $\mathrm{H}_{2}$-dependent $\mathrm{BV}$ reduction activity in anaerobically prepared, concentrated crude extracts of FTD147 ( $\triangle h y a B, \Delta h y b C, \Delta h y c E)$ transformed with phupXSL measured a low but detectable hydrogenase activity of approximately $60 \mathrm{mU} / \mathrm{mg}$ (Table 2), which is in good agreement with previously determined HupSL activity in E. coli extracts (Hartwig et al., 2015b). The phenotypically identical strain CP1170 ( $\triangle h y a B, \Delta h y b C, \Delta h y c E)$ had a background activity of $10 \mathrm{mU} / \mathrm{mg}$. Brief incubation of the extract from FTD147 + phupXSL in the presence of oxygen resulted in a reduction of the HupSL activity by $50 \%$ (Table 2).

\section{HupSL Activity in E. coli Requires a Functional Selenocysteine-Insertion Machinery}

The lack of HupSL enzyme activity after respiratory growth is reminiscent of the effects of these growth conditions on
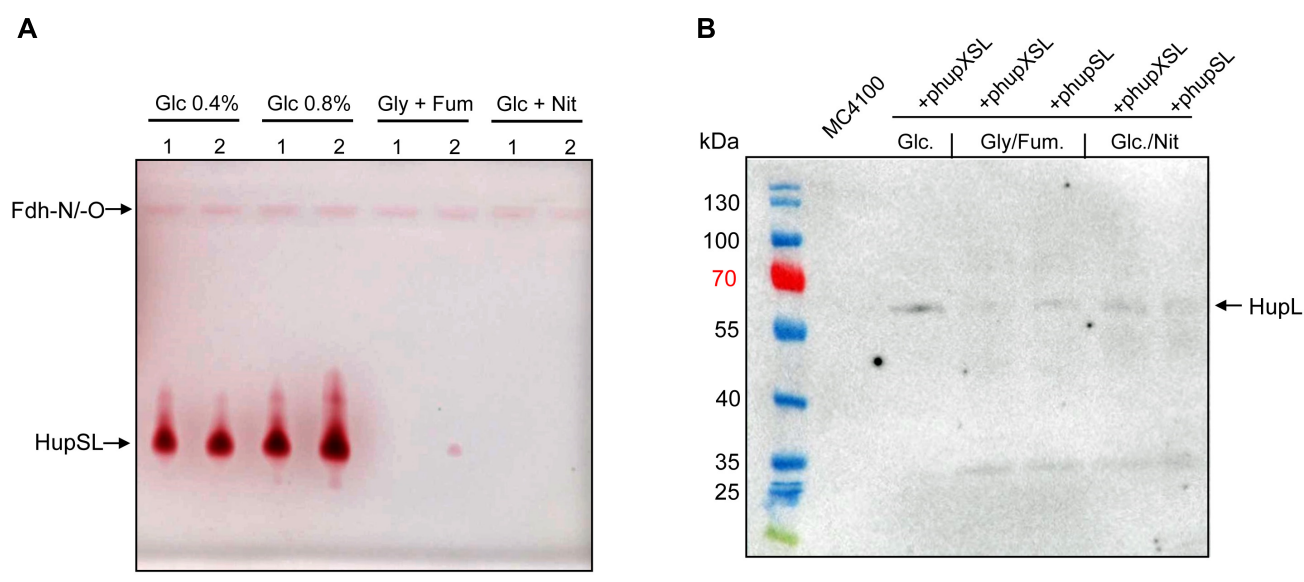

FIGURE 2 | Heterologous HupSL enzyme activity is only detectable in fermentatively growing E. coli cells. (A) Crude extracts derived from FTD147 ( $\triangle$ hyaB $\Delta$ hybC $\triangle$ hycE) transformed with plasmid phupXSL (1) or phupSL (2) after growth in M9 minimal medium with the indicated carbon sources were separated in native-PAGE and stained for hydrogenase enzyme activity, as described in the legend to Figure 1. (B) A western blot using peptide antibodies raised against HupL (Hartwig et al., 2017) is shown in which the same extracts used in part A were separated in 10\% (w/v) SDS-PAGE. The molecular mass marker proteins are presented in kDa. MC4100 was the wild type strain. 
TABLE 2 | $\mathrm{H}_{2}$ :BV oxidoreductase activities of $E$. coli strains carrying phupXSL.

\begin{tabular}{|c|c|c|}
\hline Strain & Anaerobic hydrogenase activity (mU mg protein ${ }^{-1}$ ) & Activity after $\mathrm{O}_{2}$ exposure $\left(\mathrm{mU} \mathrm{mg} \text { protein }{ }^{-1}\right)^{\mathrm{a}}$ \\
\hline CP1170 ( & $10 \pm 3^{b}$ & n.d $d^{c}$ \\
\hline FTD147 ( $\Delta$ hyaB, $\Delta$ hybC, $\Delta h y c E)+$ phupXSL & $58 \pm 18$ & $29 \pm 9$ \\
\hline FTD147 $\Delta f d n G, \Delta f d o G$ & $26 \pm 13$ & n.d. \\
\hline FTD147 $\Delta f d n G, \Delta f d o G+$ phupXSL & $59 \pm 1$ & $22 \pm 1$ \\
\hline FTD147 $\Delta f d h F$ & $4 \pm 2$ & n.d. \\
\hline FTD147 $\Delta f d h F+$ phupXSL & $11 \pm 1$ & n.d \\
\hline
\end{tabular}

${ }^{a}$ Crude extracts were exposed to air for 15 min prior to determination of hydrogenase activity. ${ }^{b}$ Assays were performed in triplicate from 3 independent growth experiments. The activity is shown together with the standard deviation. ${ }^{c}$ n.d, not determined.

appearance of E. coli Hyd-3 and Fdh-H enzyme activities (Sawers et al., 1985), with the exception that the effect on synthesis of the E. coli enzymes is at the transcriptional level due to depletion of the regulatory metabolite formate (Rossmann et al., 1991). Due to the fact that HupSL is naturally associated with a formate dehydrogenase-like enzyme, OmeAB (Kublik et al., 2016; Hartwig et al., 2017), we wished to examine the influence of the Fdh-O and Fdh-N enzymes, which are phylogenetically related to OmeAB, on HupSL enzyme activity. Initially, we introduced into strain FTD147 a mutation in the selC gene, which encodes the selenocysteinyl-tRNA Sec $_{\text {necessary }}$ for translation of special UGA codons as selenocyteine, and which, when deleted, renders all Fdhs inactive (Leinfelder et al., 1988). This would provide information on whether HupSL enzyme activity was influenced by defects in formate metabolism. The left panel shown in Figure 3 shows a control for HupSL activity revealing that is was readily detectable in strain FTD150 ( $\Delta h y a B \Delta h y b C \Delta h y c E \Delta h y f G)$, which is identical to strain FTD147 with the exception that the gene encoding the catalytic subunit of Hyd-4 (Andrews et al., 1997) is also deleted (Redwood et al., 2008). Thus, both FTD150 and FTD147

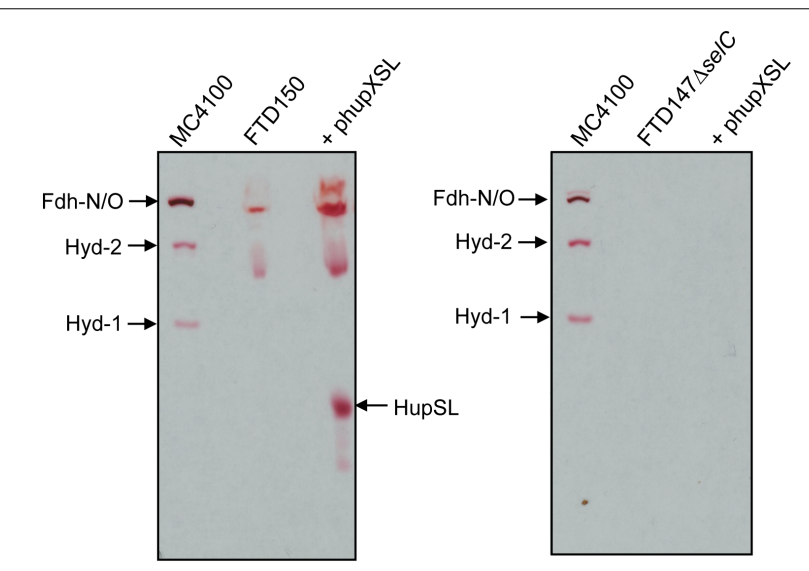

FIGURE 3 | Appearance of HupSL enzyme activity is dependent on a functional selenocysteine biosynthetic apparatus. Crude extracts (100 $\mu \mathrm{g}$ of protein, except M4100 where $30 \mu \mathrm{g}$ of protein were applied) of the indicated strains were separated in native-PAGE and stained for hydrogenase enzyme activity as described in the legend to Figure 1. Strains used include: MC4100 (wild type); FTD150 ( $\Delta$ hyaB $\Delta$ hybC $\Delta$ hycE $\Delta$ hyfG); FTD147 $\Delta$ se/C ( $\Delta$ hyaB $\Delta$ hybC $\Delta$ hycE $\Delta$ se/C). yield an identical phenotype with regard to the heterologous HupSL activity (see also below). Analysis of an extract of the FTD147 $\Delta$ selC mutant revealed that no HupSL activity was detectable (Figure 3 right panel). The lack of selC was confirmed by the absence of the $\mathrm{H}_{2}$ : $\mathrm{BV}$ oxidoreductase activity associated with Fdh-N/O in the strain (Soboh et al., 2011). This result confirms that HupSL enzyme activity is linked to formate metabolism, most likely through one of the formate dehydrogenases (Fdh) the bacterium synthesizes under anaerobic conditions. Introduction of a mutation in $s e l B$, which encodes the special translation factor required to decode the UGA codon as selenocysteine (Forchhammer et al., 1989), into FTD150 also revealed a similar lack of HupSL activity (data not shown), confirming that the phenotype was due to a lack of selenocysteine incorporation.

\section{In-Gel HupSL Activity Depends on the Fdh-H Enzyme}

Dependence on the selenocysteine biosynthetic machinery for appearance of HupSL enzyme activity suggests an involvement of one or more of the three Fdhs present in E. coli. To determine which of the three Fdhs is required for the appearance of heterologous HupSL activity, we constructed a series of strains (see Table 1) lacking one or more of the genes encoding the catalytic subunit of FdnG (of FdhN), FdoG (of Fdh-O), or FdhF (of Fdh-H) (Pinske and Sawers, 2016; Figure 4). Strain FTD150, which lacked all four hydrogenases and the quadruple and quintuple mutants of FTD147, which lacked Hyd-1, Hyd-2, Hyd-3 as well as either or both respiratory Fdhs (Fdh-N and Fdh-O), retained fully active HupSL (Figure 4). Hydrogenase activity in extracts derived from FTD $147 \Delta f d n G \Delta f d o G$ with plasmid phupXSL was approximately $60 \mathrm{mU}$, while the strain without plasmid had approximately half this activity (Table 2). Exposure of the crude extract from FTD $147 \Delta f d n G \Delta f d o G$ transformed with phupXSL to air resulted in a similar $50-60 \%$ reduction in hydrogenase activity as was observed with FTD147 containing phupXSL (Table 2). This result confirms that HupSL is oxygen-labile (Hartwig et al., 2015b).

The only strains that lacked a detectable HupSL enzyme activity band were those that lacked the $f d h F$ gene, which encodes the Fdh-H component of the FHL complex (Figure 4). Assay of hydrogenase activity in extracts derived from FTD147 $\Delta f h F+$ phupXSL failed to show HupSL-dependent hydrogenase activity 

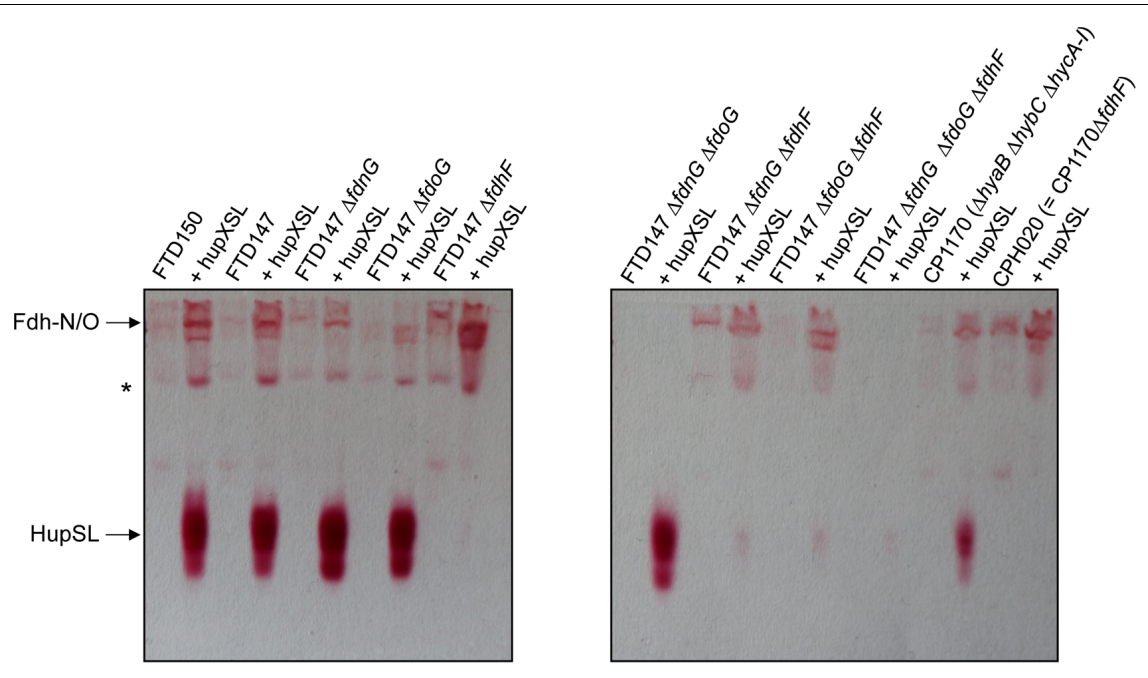

FIGURE 4 | Only strains lacking Fdh-H, the product of the $f d h F$ gene, failed to show HupSL enzyme activity. Crude extracts (90 $\mu \mathrm{g}$ of protein) of the indicated strains were separated in native-PAGE and stained for hydrogenase enzyme activity as described in the legend to Figure 1. The asterisk denotes a fast-migrating form of the Fdh-O enzyme (Hartwig et al., 2015a). Strains used include: FTD147 ( $\Delta$ hyaB $\Delta$ hybC $\Delta$ hycE), plus its deletion derivatives; FTD150 ( $\Delta$ hyaB $\Delta$ hybC $\Delta$ hycE $\Delta$ hyfG);

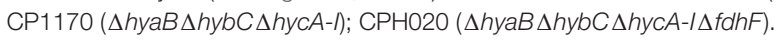

(Table 2). These findings indicate that for full HupSL activity to be manifested, an active Fdh-H enzyme is required.

In order to determine what the link between the appearance of HupSL enzyme activity and Fdh-H might be, we first performed a western blot using anti-HupX antibodies and with crude extracts derived from some of the strains shown in Figure 4. Surprisingly, HupX was only detectable in extracts of strains lacking $f d h F$, which encodes the Fdh-H enzyme, and, as expected, only in those strains carrying the phupXSL plasmid (Figure 5). This suggests that when Fdh-H was absent, HupX was stably synthesized and

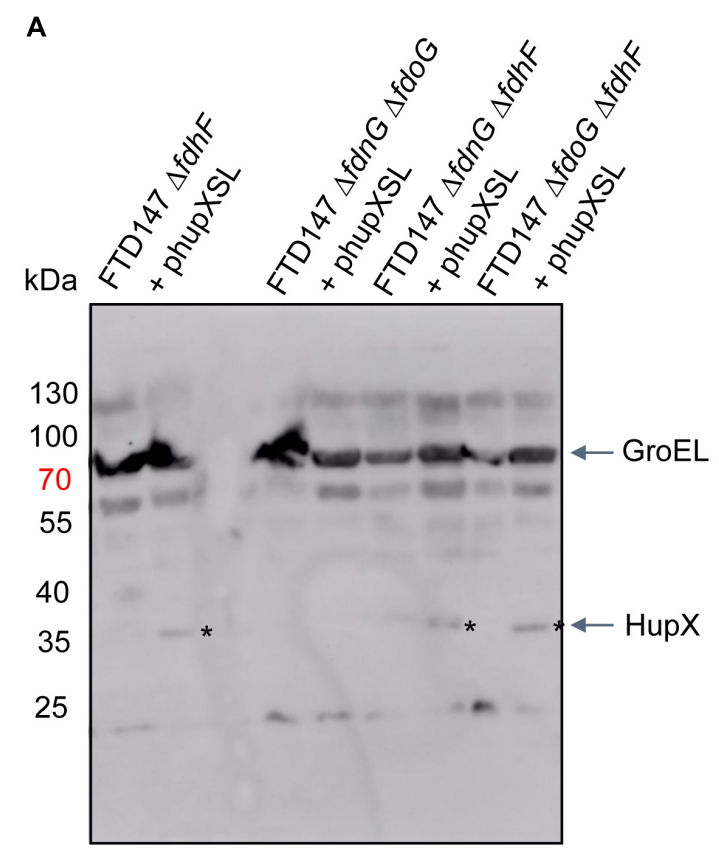

B

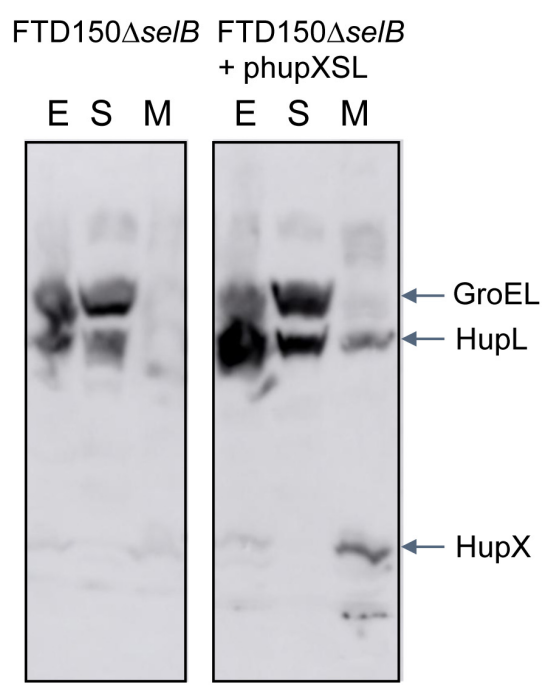

FIGURE 5 | HupX is only detectable in extracts of strains lacking the fdhF gene. Western blots using peptide antibodies raised against HupX or HupL are shown in which $50 \mu \mathrm{g}$ of crude extracts (A) or subcellular fractions (B) were separated in 10\% (w/v) SDS-PAGE. The migration positions of molecular mass marker proteins are shown in $\mathrm{kDa}$. The strong signal migrating around $70 \mathrm{kDa}$ represents GroEL, which cross-reacted with the HupX antibodies, and acted as a protein loading control. The asterisks denote the HupX polypeptide. In (B), the letters E, S, and M represent crude extract, soluble cytoplasmic fraction and membrane fraction, respectively. Strain used include: deletion derivatives of FTD147 ( $\triangle$ hyaB $\Delta$ hybC $\Delta$ hycE); and deletion derivatives of FTD150 ( $\triangle$ hyaB $\Delta h y b C \Delta h y c E \Delta h y f G)$. 
when Fdh-H was present in the cells, HupX became unstable and was presumably degraded.

\section{The Influence of Fdh-H on HupSL Activity Is Indirect via the Ferredoxin-Like Proteins HupX and HycB}

To examine whether HupX might influence HupSL activity, plasmids phupSL and phupXSL, both encoding HupL, HupS and the endoprotease HoxM, but only the latter also encoding HupX (Figure 1A), were introduced into strains FTD147 and FTD147 $\triangle d h F$ and enzyme activity was compared after anaerobic growth with glucose (Figure 6A). The products of both plasmids in strain FTD $147 \Delta f d h F$ showed a strongly reduced activity of HupSL compared with the $f d h F^{+}$strain FTD147. This result indicates that the dependence on Fdh-H for HupSL activity was retained in the absence of HupX.

A recent study in $E$. coli identified a flexible interaction network of ferredoxin-like proteins with $\mathrm{Fdh}-\mathrm{H}$, including its small, electron-transferring subunit, $\mathrm{HycB}$ (Pinske, 2018). HycB and HupX belong to this family but share only $24 \%$ amino acid identity (38\% similarity) in a MuscleWS alignment. The HupX protein, however, cannot functionally replace $\mathrm{HycB}$ in formate-dependent $\mathrm{BV}$ reduction (data not shown). Due to the link between HupSL activity and Fdh-H demonstrated above, we therefore examined whether the presence of $\mathrm{HycB}$ influenced HupSL's ability to reduce BV. To do this, we analyzed HupSL activity in strain CP1170 ( $\Delta$ hyaB, $\Delta h y b C, \Delta h y c A-I)$, which is similar to FTD147 ( $\triangle h y a B, \Delta h y b C, \Delta h y c E)$ with the exception that the complete hyc operon is deleted in
CP1170, rather than only the hycE gene (Table 1). Introduction of plasmid phupXSL into CP1170 and its $\Delta f d h F$ derivative CPH020 ( $\Delta h y a B \Delta h y b C \Delta h y c A-I \Delta f d h F$ ) (Table 1), revealed that the dependence on Fdh-H for HupSL activity was retained (Figure 4, right side of right panel). However, introduction of plasmid phupSL (lacking hupX) into CPH020 (CP1170 $\Delta f d h F)$ revealed that HupSL activity in native PAGE was no longer reduced in the absence of Fdh-H (Figure 6B, lane 1, right panel). Introduction of phupSL into strain CPH008 ( $\triangle$ hycA$I, \Delta f d h F)$ in which Hyd-1 and Hyd-2 are still active, but all structural components of the FHL complex are missing, demonstrated that HupSL activity was retained, and even slightly more intense (Figure 6B, lane 2 left panel). As a final control, we analyzed HupSL activity after introduction of phupSL into strain RT2 ( $\Delta h y a B \Delta h y b C \Delta h y c A-I \Delta f d h E \Delta p f l A)$, which lacks Hyd-1, Hyd-2 and Hyd-3, as well as Fdh-N/O (through the fdhE mutation; Mandrand-Berthelot et al., 1988; Lüke et al., 2008) and the formate-inducible Fdh-H, due to the lack of active PflB (due to the $p f l A$ mutation), which is required for formate production (Sawers and Böck, 1988; Rossmann et al., 1991). This strain was chosen because it uses a different combination of mutations to generate the same phenotype, i.e., no hydrogenase or formate dehydrogenase enzymes, and no $\mathrm{HycB}$ protein. HupSL activity was also retained in this genetic background confirming that strains devoid of HupX and $\mathrm{HycB}$ exhibit HupSL- and $\mathrm{H}_{2}$-dependent reduction of BV (Figure 6B, lane 1).

Surprisingly, however, when phupSL was introduced into strain RM220 ( $\triangle p f l A B)$, which generates considerably reduced levels of formate under respiratory conditions (Suppmann and
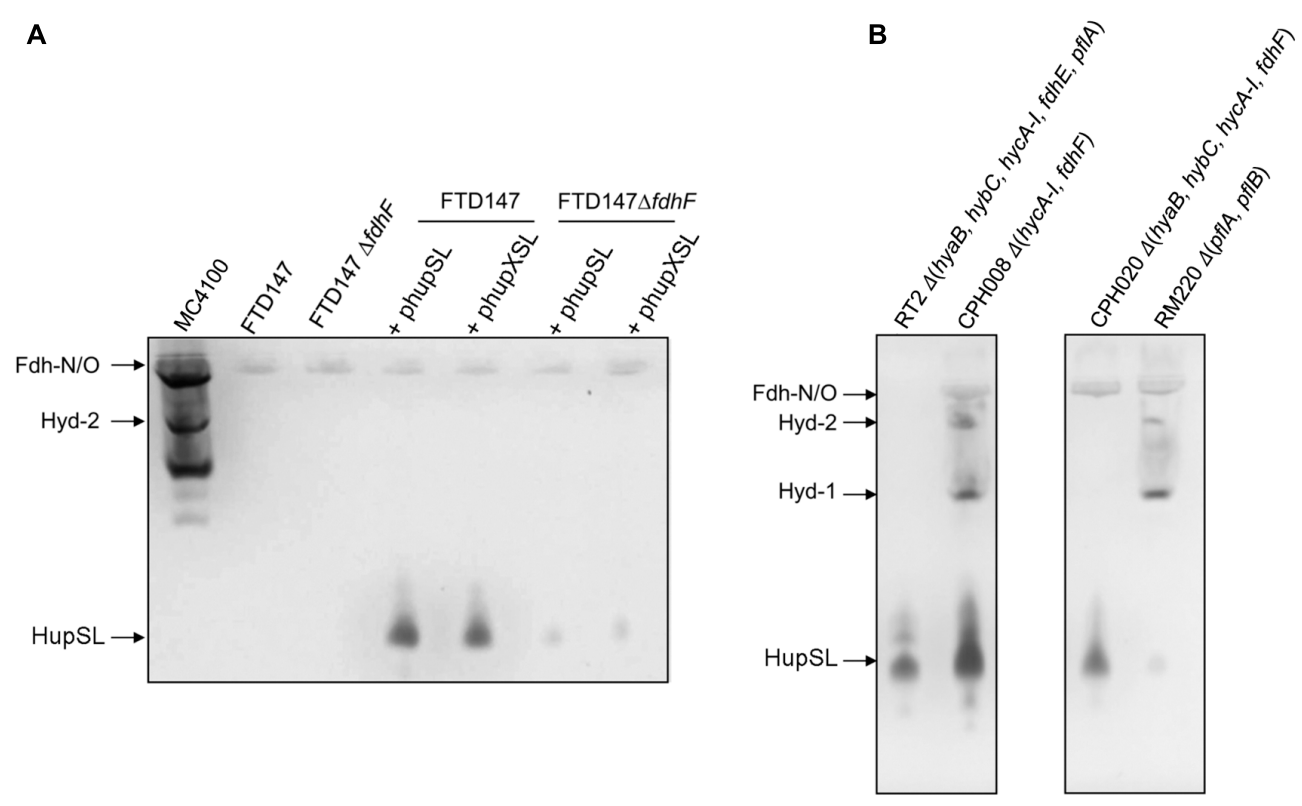

FIGURE 6 | Deletion of the hycA-I operon restores HupSL activity in a fdhF mutant. (A) Crude extracts derived (approximately 60 $\mu \mathrm{g}$ of protein) from the indicated strains after growth in M9 minimal medium with $0.8 \%(\mathrm{w} / \mathrm{V})$ glucose were separated in native-PAGE and stained for hydrogenase enzyme activity, as described in the legend to Figure 1. In (B), strains were transformed with plasmid phupSL. The migration positions of the respective hydrogen-oxidizing enzymes are indicated. Strain used include: MC4100 represents wild type; FTD147 ( hyaB $\Delta$ hybC $\Delta h y c E)$; FTD147 $\Delta f d h F ;$ RT2 ( $\Delta h y a B \Delta h y b C \Delta h y c A-I \Delta f d h E \Delta p f I A) ;$ CPH008 ( $\Delta h y c A-I$

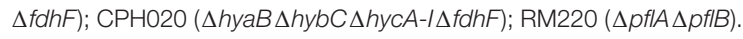


Sawers, 1994), and thus expresses only low levels of the formateinducible hyc operon (Rossmann et al., 1991), the anticipated high activity of HupSL was not observed (Figure 6B, lane 4). This result indicates that even the low levels of $\mathrm{HycB}$ produced in this strain (Rossmann et al., 1991) are likely sufficient to inhibit HupSL activity.

\section{DISCUSSION}

In this study, we analyzed how the fermentative metabolism of the E. coli host influenced the redox dye-reducing activity of a heterologously synthesized hydrogen-uptake [NiFe]hydrogenase from the Chloroflexi phylum. We had previously demonstrated that the host's Hyp-maturation machinery was capable of recognizing the large subunit precursor pre-HupL and of successfully introducing the bimetallic [NiFe]-cofactor, generating active enzyme (Hartwig et al., 2015b). We also showed in that particular study that the HupSL hydrogenase had $\mathrm{H}_{2}$ :BV oxidoreductase activity, which could be identified after anaerobic native-PAGE. Here we made the surprising discovery that the appearance of this HupSL enzyme activity was apparently dependent on whether the host's Fdh-H enzyme was synthesized or not. Under conditions favoring synthesis of Fdh-H, HupSL activity was observed, while in strains unable to synthesize Fdh-H, due to a deletion of the $f d h F$ structural gene or the selenocysteine insertion machinery, no, or substantially reduced, activity was detected. Notably, however, this lack of enzyme activity did not result from a lack of synthesis of the HupSL enzyme, but rather appears to be due to an inactivation of the enzyme.

A recent study revealed that $\mathrm{Fdh}-\mathrm{H}$ interacts with at least three electron-transferring small subunits, all of which belong to the ferredoxin-like family of electron-transfer proteins and possibly facilitate the coupling of Fdh-H with different enzyme complexes (Pinske, 2018). We currently interpret our data to indicate that the apparent dependence on Fdh-H for HupSL activity is, in fact, indirect and likely due to $\mathrm{Fdh}-\mathrm{H}$ sequestering these small subunits, in particular $\mathrm{HycB}$ of the FHL complex. Notably, the HupX protein, which is presumed to mediate electron transfer within the Hup-Ome-Rdh supercomplex in the natural host D. mccartyi (Schubert et al., 2018), also belongs to the ferredoxinlike superfamily and this protein's ability to interact with the HupSL heterodimer also appears to be influenced by the presence of Fdh-H. If Fdh-H is either genotypically or phenotypically (e.g., through strongly reduced formate synthesis; Rossmann et al., 1991) absent, the ferredoxin-like proteins HupX or HycB remain consequently unbound within the cell. We suggest that if HupX is also absent, $\mathrm{HycB}$ can transiently interact with or modulate the HupSL enzyme within the cell prior to separation in the nativePAGE, rendering the enzyme inactive. This inactivity could result from a loss of the ability of the heterodimer to reduce or interact with $\mathrm{BV}$ in the presence of $\mathrm{H}_{2}$. Alternatively, these ferredoxinlike proteins might act by sequestering the HupSL complex resulting in an inactive complex in the native-PAGE; or indeed a combination of both effects might be the cause (Figure 7). The consequence would be that HupSL activity becomes visible in the

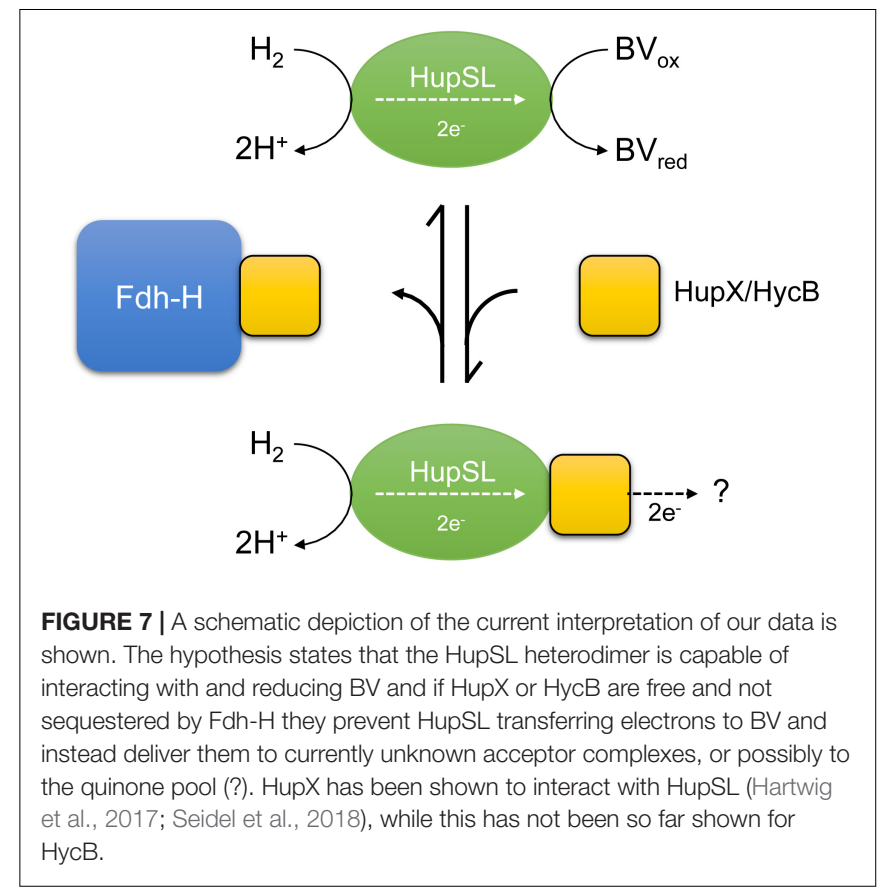

absence of $\mathrm{HycB}$ despite simultaneous absence of Fdh-H. The redox-potentials of the ferredoxin-like proteins have not yet been determined.

The model shown in Figure 7 presents a working hypothesis for how we currently interpret our data. Under fermentative growth conditions Fdh-H is present and is available to interact with HycB. The presence of Fdh-H also prevents HupX interacting with HupSL, possibly forming an interaction with HupX, allowing the HupSL heterodimer to interact with and reduce $\mathrm{BV}$.

How does an ability to interact with HupX, and possibly $\mathrm{HycB}$, interfere with electron transfer to BV by HupSL? The redox dye BV can accept electrons directly from the electron-transferring subunit HupS, as evidenced by the fact that deletion of the hupX gene does not significantly affect $\mathrm{H}_{2}$-dependent reduction of $\mathrm{BV}$ by HupSL. Moreover, previous mass spectrometric analysis of the Hup activity band isolated after native-PAGE revealed mainly HupL and HupS to be present, suggesting that HupX's interaction with the heterodimer is weak or transient (Hartwig et al., 2015b). Moreover, in the natural host D. mccartyi, HupX preferentially associates with $\mathrm{OmeAB}$, the formate dehydrogenase homolog, and reductive dehydrogenases rather than with HupSL (Hartwig et al., 2015b; Seidel et al., 2018; Dragomirova and Sawers, unpublished observations), which supports the suggestion that the affinity of HupX for HupSL is low. We also observed using antibodies raised against HupX that it is only readily detectable in the membrane fraction of cells that lack Fdh-H, suggesting that when Fdh-H is present it is more readily degraded. How this apparent degradation is controlled is currently unclear.

The HupS subunit encodes a functional Tat-signal peptide allowing its transport across the cytoplasmic membrane (Hartwig et al., 2015b). Together with HupX, the HupSL complex could be sufficiently anchored in the membrane to transfer the electrons 
derived from oxidation of $\mathrm{H}_{2}$ to the quinone pool, which is also the function of another HupX homolog, the HybA protein of E. coli Hyd-2 (Pinske et al., 2015; Beaton et al., 2018).

Support for the oxidative inactivation of HupSL was provided by the demonstration of inactivation of the enzyme complex after growth of the E. coli host under respiratory conditions, with either $\mathrm{O}_{2}\left(E^{\mathrm{O} \prime}=+830 \mathrm{mV}\right), \mathrm{NO}_{3}{ }^{-}$ $\left(E^{\mathrm{o}^{\prime}}=+420 \mathrm{mV}\right)$, or fumarate $\left(E^{\mathrm{o} \prime}=0 \mathrm{mV}\right)$ as electron acceptor. We have only been able to detect HupSL activity after fermentative growth $\left(E^{\mathrm{o} \prime}=-415 \mathrm{mV}\right)$, strongly suggesting that the enzyme retains activity only under strongly reducing conditions, which are also likely to be those prevailing in the environmental conditions where D. mccartyi is found (Löffler et al., 2013).

These studies thus provide a platform to study how heterologously synthesized hydrogenases can be integrated into the host's anaerobic metabolism. Clearly, this work is at an early stage but one of the next steps will be to examine whether electrons derived from $\mathrm{H}_{2}$ oxidation can be coupled to reduction of E. coli-typical electron acceptors, e.g., fumarate. Initial studies examining hydrogen-driven fumarate reduction by HupXLS yielded first indications that a weak, but unfortunately so far irreproducible, activity was detectable (Schwoch et al., unpublished data). Optimization of Hup enzyme synthesis and membrane integration will likely be required for this approach to be fruitful.

Because D. mccartyi species are not amenable to large-scale biochemical analysis, and are currently genetically intractable, using the E. coli Hyd- and Fdh-negative host strains developed

\section{REFERENCES}

Andrews, S. C., Berks, B. C., McClay, J., Ambler, A., Quail, M. A., Golby, P., et al. (1997). A 12-cistron Escherichia coli operon (hyf) encoding a putative protontranslocating formate hydrogenlyase system. Microbiology 143, 3633-3647. doi: 10.1099/00221287-143-11-3633

Baba, T., Ara, T., Hasegawa, M., Takai, Y., Okumura, Y., Baba, M., et al. (2006). Construction of Escherichia coli K-12 in-frame, single-gene knockout mutants: the Keio collection. Mol. Syst. Biol. 2:0008. doi: 10.1038/msb4100050

Ballantine, S. P., and Boxer, D. H. (1985). Nickel-containing hydrogenase isoenzymes from anaerobically grown Escherichia coli K-12. J. Bacteriol. 163, 454-459.

Beaton, S. E., Evans, R. M., Finney, A. J., Lamont, C. M., Armstrong, F. A., Sargent, F., et al. (2018). The structure of hydrogenase-2 from Escherichia coli: implications for H2-driven proton pumping. Biochem. J. 475, 1353-1370. doi: 10.1042/BCJ20180053

Begg, Y., Whyte, J., and Haddock, B. A. (1977). The identification of mutants of Escherichia coli deficient in formate dehydrogenase and nitrate reductase activities using dye indicator plates. FEMS Microbiol. Lett. 2, 47-50. doi: 10. 1111/j.1574-6968.1977.tb00905.x

Böck, A., King, P., Blokesch, M., and Posewitz, M. (2006). Maturation of hydrogenases. Adv. Microb. Physiol. 51, 1-71. doi: 10.1016/S0065-2911(06) 51001-X

Casadaban, M. J. (1976). Transposition and fusion of the lac genes to selected promoters in Escherichia coli using bacteriophage lambda and Mu. J. Mol. Biol. 104, 541-555. doi: 10.1016/0022-2836(76)90119-4

Cherepanov, P. P., and Wackernagel, W. (1995). Gene disruption in Escherichia coli: TcR and KmR cassettes with the option of Flp-catalyzed excision of the antibiotic-resistance determinant. Gene 158, 9-14. doi: 10.1016/0378-1119(95) 00193-A here will provide a means of studying the biochemical mechanism(s) underlying the loss of HupSL activity in response to oxidizing redox conditions and whether this effect is linked to a particular iron-sulfur cluster, or clusters, in HupS, or whether the bimetallic cofactor in HupL is the target of irreversible inactivation. A recent study by Hartmann et al. (2018) indicates that, at least for certain [NiFe]-hydrogenases, the $\mathrm{NiFe}(\mathrm{CN})_{2} \mathrm{CO}$ cofactor is not sensitive to oxidative conditions, suggesting that it might indeed be the electron-transfer pathway that is disrupted by non-reducing redox potentials.

\section{AUTHOR CONTRIBUTIONS}

ND, PR, SS, SH, and CP designed and performed the experiments and analyzed the data. CP and RS conceived the study, interpreted the data and drafted the manuscript. All authors read and approved the manuscript.

\section{FUNDING}

This work was funded by the Deutsche Forschungsgemeinschaft as part of the research unit FOR1530: "Anaerobic Biological Dehalogenation: Organisms, Biochemistry, and (Eco)physiology" granted to RS and by the priority program SPP1927 "Iron Sulfur for Life" granted to both CP and RS. We acknowledge the financial support of the Open Access Publication Fund of the Martin Luther University Halle-Wittenberg.

Dubini, A., Pye, R., Jack, R., Palmer, T., and Sargent, F. (2002). How bacteria get energy from hydrogen: a genetic analysis of periplasmic hydrogen oxidation in Escherichia coli. Int. J. Hydrogen Energy 27, 1413-1420. doi: 10.1016/S03603199(02)00112-X

Fincker, M., and Spormann, A. M. (2017). Biochemistry of catabolic reductive dehalogenation. Annu. Rev. Biochem. 86, 357-386. doi: 10.1146/annurevbiochem-061516-044829

Forchhammer, K., Leinfelder, W., and Böck, A. (1989). Identification of a novel translation factor necessary for the incorporation of selenocysteine into protein. Nature 342, 453-456. doi: 10.1038/342453a0

Hartmann, S., Frielingsdorf, S., Ciaccafava, A., Lorent, C., Fritsch, J., Siebert, E., et al. (2018). O2-tolerant H2 activation by a solitary large subunit of a [NiFe] hydrogenase. Biochemistry 57, 5339-5349. doi: 10.1021/acs.biochem.8b00760

Hartwig, S., Dragomirova, N., Kublik, A., Türkowsky, D., von Bergen, M., Lechner, U., et al. (2017). A H2-oxidizing, 1,2,3-trichlorobenzene-reducing multienzyme complex isolated from the obligately organohalide-respiring bacterium Dehalococcoides mccartyi strain CBDB1. Environ. Microbiol. Rep. 9, 618-625. doi: 10.1111/1758-2229.12560

Hartwig, S., Pinske, P., and Sawers, R. G. (2015a). Chromogenic assessment of the three molybdo-selenoprotein formate dehydrogenases in Escherichia coli. Biochem. Biophys. Rep. 1, 62-67. doi: 10.1016/j.bbrep.2015.03.006

Hartwig, S., Thomas, C., Krumova, N., Quitzke, V., Türkowsky, D., Jehmlich, N., et al. (2015b). Heterologous complementation studies in Escherichia coli with the Hyp accessory protein machinery from Chloroflexi provide insight into [NiFe]-hydrogenase large subunit recognition by the HypC protein family. Microbiology 161, 2204-2219. doi: 10.1099/mic.0.000177

Hormann, K., and Andreesen, J. R. (1989). Reductive cleavage of sarcosine and betaine by Eubaterium acidaminophilum via enzyme systems different from glycine reductase. Arch. Microbiol. 153, 50-59. doi: 10.1007/BF0027 7541 
Kaiser, M., and Sawers, G. (1994). Pyruvate formate-lyase is not essential for nitrate respiration by Escherichia coli. FEMS Microbiol. Lett. 117, 163-168. doi: 10.1111/j.1574-6968.1994.tb06759.x

Kube, M., Beck, A., Zinder, S. H., Kuhl, H., Reinhardt, R., and Adrian, L. (2005). Genome sequence of the chlorinated compound-respiring bacterium Dehalococcoides species strain CBDB1. Nat. Biotechnol. 23, 1269-1273. doi: $10.1038 /$ nbt1131

Kublik, A., Deobald, D., Hartwig, S., Schiffmann, C. L., Andrades, A., von Bergen, M., et al. (2016). Identification of a multiprotein reductive dehalogenase complex in Dehalococcoides mccartyi strain CBDB1 suggests a proteindependent respiratory electron transport chain obviating quinone involvement. Environ. Microbiol. 18, 3044-3056. doi: 10.1111/1462-2920.13200

Laemmli, U. (1970). Cleavage of structural proteins during the assembly of the head of bacteriophage T4. Nature 227, 680-685. doi: 10.1038/227680a0

Leinfelder, W., Zehelein, E., Mandrand-Berthelot, M.-A., and Böck, A. (1988). Gene for a novel tRNA species that accepts L-serine and cotranslationally inserts selenocysteine. Nature 331, 723-725. doi: 10.1038/331723a0

Löffler, F. E., Yan, J., Ritalahti, K. M., Adrian, L., Edwards, E. A., Konstantinidis, K. T., et al. (2013). Dehalococcoides mccartyi gen. nov., sp. nov., obligately organohalide-respiring anaerobic bacteria relevant to halogen cycling and bioremediation, belong to a novel bacterial class, Dehalococcoidia classis nov., order Dehalococcoidales ord. nov. and family Dehalococcoidaceae fam. nov., within the phylum Chloroflexi. Int. J. Syst. Evol. Microbiol. 63, 625-635. doi: 10.1099/ijs.0.034926-0

Lowry, O., Rosebrough, N., Farr, A., and Randall, R. (1951). Protein measurement with the Folin phenol reagent. J. Biol. Chem. 193, 265-275.

Lüke, I., Butland, G., Moore, K., Buchanan, G., Lyall, V., Fairhurst, S. A., et al. (2008). Biosynthesis of the respiratory formate dehydrogenases from Escherichia coli: characterization of the FdhE protein. Arch. Microbiol. 90, 685-696. doi: 10.1007/s00203-008-0420-4

Mandrand-Berthelot, M.-A., Couchoux-Luthaud, G., Santini, C.-L., and Giordano, G. (1988). Mutants of Escherichia coli specifically defective in respiratory formate dehydrogenase activity. J. Gen. Microbiol. 134, 3129-3139.

Miller, J. (1972). Experiments in Molecular Genetics. Cold Spring Harbor, NY: Cold Spring Harbor Laboratory.

Pinske, C. (2018). The ferredoxin-like proteins HydN and YsaA enhance redox dye-linked activity of the formate dehydrogenase $\mathrm{H}$ component of the formate hydrogenlyase complex. Front. Microbiol. 9:1238. doi: 10.3389/fmicb.2018. 01238

Pinske, C., Jaroschinsky, M., Linek, S., Kelly, C. L., Sargent, F., and Sawers, R. G. (2015). Physiology and bioenergetics of [NiFe]-hydrogenase 2-catalyzed H2consuming and H2-producing reactions in Escherichia coli. J. Bacteriol. 197, 296-306. doi: 10.1128/JB.02335-14

Pinske, C., Jaroschinsky, M., Sargent, F., and Sawers, G. (2012). Zymographic differentiation of [NiFe]-hydrogenases 1, 2 and 3 of Escherichia coli K-12. BMC Microbiol. 12:134. doi: 10.1186/1471-2180-12-134

Pinske, C., Krüger, S., Soboh, B., Ihling, C., Kuhns, M., Braussemann, M., et al. (2011). Efficient electron transfer from hydrogen to benzyl viologen by the [NiFe]-hydrogenases of Escherichia coli is dependent on the coexpression of the iron-sulfur cluster-containing small subunit. Arch. Microbiol. 193, 893-903. doi: 10.1007/s00203-011-0726-5

Pinske, C., and Sargent, F. (2016). Exploring the directionality of Escherichia coli formate hydrogenlyase: a membrane-bound enzyme capable of fixing carbon dioxide to organic acid. Microbiologyopen 9:1238. doi: 10.1002/mbo 3.365

Pinske, C., and Sawers, R. G. (2016). Anaerobic formate and hydrogen metabolism. EcoSal Plus. doi: 10.1128/ecosalplus.ESP-0011-2016
Redwood, M. D., Mikheenko, I., Sargent, F., and Macaskie, L. (2008). Dissecting the roles of Escherichia coli hydrogenases in biohydrogen production. FEMS Microbiol. Lett. 278, 48-55. doi: 10.1111/j.1574-6968.2007.00966.x

Rossmann, R., Sawers, G., and Böck, A. (1991). Mechanism of regulation of the formate-hydrogenlyase pathway by oxygen, nitrate and $\mathrm{pH}$ : definition of the formate regulon. Mol. Microbiol. 5, 2807-2814. doi: 10.1111/j.1365-2958.1991. tb01989.x

Sambrook, J., Fritsch, E. F., and Maniatis, T. (1989). Molecular Cloning: A Laboratory Manual, 2nd Edn. Cold Spring Harbor, NY: Cold Spring Harbor Laboratory.

Sargent, F., Ballantine, S. P., Rugman, P. A., Palmer, T., and Boxer, D. H. (1998). Reassignment of the gene encoding the Escherichia coli hydrogenase 2 small subunit: identification of a soluble precursor of the small subunit in a hypB mutant. Eur. J. Biochem. 255, 746-754. doi: 10.1046/j.1432-1327.1998.2550 746.x

Sawers, G., and Böck, A. (1988). Anaerobic regulation of pyruvate formate-lyase from Escherichia coli K-12. J. Bacteriol. 170, 5330-5336. doi: 10.1128/jb.170.11. 5330-5336.1988

Sawers, R. G., Ballantine, S. P., and Boxer, D. H. (1985). Differential expression of hydrogenase isoenzymes in Escherichia coli K-12: evidence for a third isoenzyme. J. Bacteriol. 164, 1324-1331.

Schipp, C. J., Marco-Urrea, E., Kublik, A., Seifert, J., and Adrian, L. (2013). Organic cofactors in the metabolism of Dehalococcoides mccartyi strains. Philos. Trans. R. Soc. Lond. B Biol. Sci. 368:20120321. doi: 10.1098/rstb.2012. 0321

Schubert, T., Adrian, L., Sawers, R. G., and Diekert, G. (2018). Organohalide respiratory chains: composition, topology, and key enzymes. FEMS Microbiol. Ecol. 94:4. doi: 10.1093/femsec/fiy035

Seidel, K., Kühnert, J., and Adrian, L. (2018). The complexome of Dehalococcoides mccartyi reveals its organohalide respiration-complex is modular. Front. Microbiol. 9:1130. doi: 10.3389/fmicb.2018. 01130

Soboh, B., Pinske, C., Kuhns, M., Waclawek, M., Ihling, C., Trchounian, K., et al. (2011). The respiratory molybdo-selenoprotein formate dehydrogenases of Escherichia coli have hydrogen: benzyl viologen oxidoreductase activity. BMC Microbiol. 11:173. doi: 10.1186/1471-2180-11-173

Suppmann, B., and Sawers, G. (1994). Isolation and characterisation of hypophosphite-resistant mutants of Escherichia coli: identification of the FocA protein, encoded by the $p f l$ operon, as a putative formate transporter. Mol. Microbiol. 11, 965-982. doi: 10.1111/j.1365-2958.1994.tb00 375.x

Towbin, H., Staehelin, T., and Gordon, J. (1979). Electrophoretic transfer of proteins from polyacrylamide gels to nitrocellulose sheets: procedure and some applications. Proc. Natl. Acad. Sci. U.S.A. 76, 4350-4354. doi: 10.1073/pnas.76. 9.4350

Conflict of Interest Statement: The authors declare that the research was conducted in the absence of any commercial or financial relationships that could be construed as a potential conflict of interest.

Copyright ( 2018 Dragomirova, Rothe, Schwoch, Hartwig, Pinske and Sawers. This is an open-access article distributed under the terms of the Creative Commons Attribution License (CC BY). The use, distribution or reproduction in other forums is permitted, provided the original author(s) and the copyright owner(s) are credited and that the original publication in this journal is cited, in accordance with accepted academic practice. No use, distribution or reproduction is permitted which does not comply with these terms. 